\title{
Front Matter: Volume 10110
}

, "Front Matter: Volume 10110," Proc. SPIE 10110, Photonic Instrumentation Engineering IV, 1011001 (31 March 2017); doi: 10.1117/12.2276081

SPIE. Event: SPIE OPTO, 2017, San Francisco, California, United States 


\title{
PROCEEDINGS OF SPIE
}

\section{Photonic Instrumentation Engineering IV}

\author{
Yakov G. Soskind \\ Craig Olson \\ Editors
}

31 January-2 February 2017

San Francisco, California, United States

Sponsored and Published by

SPIE 
The papers included in this volume were part of the technical conference cited on the cover and title page. Papers were selected and subject to review by the editors and conference program committee. Some conference presentations may not be available for publication. The papers published in these proceedings reflect the work and thoughts of the authors and are published herein as submitted. The publisher is not responsible for the validity of the information or for any outcomes resulting from reliance thereon.

Please use the following format to cite material from this book:

Author(s), "Title of Paper," in Photonic Instrumentation Engineering IV, edited by Yakov G. Soskind, Craig Olson, Proceedings of SPIE Vol. 10110 (SPIE, Bellingham, WA, 2017) Seven-digit Article CID Number.

ISSN: 0277-786X

ISSN: 1996-756X (electronic)

ISBN: 9781510606616

ISBN: 9781510606623 (electronic)

Published by

SPIE

P.O. Box 10, Bellingham, Washington 98227-0010 USA

Telephone +1 3606763290 (Pacific Time) · Fax +1 3606471445

SPIE.org

Copyright (C) 2017, Society of Photo-Optical Instrumentation Engineers.

Copying of material in this book for internal or personal use, or for the internal or personal use of specific clients, beyond the fair use provisions granted by the U.S. Copyright Law is authorized by SPIE subject to payment of copying fees. The Transactional Reporting Service base fee for this volume is $\$ 18.00$ per article (or portion thereof), which should be paid directly to the Copyright Clearance Center (CCC), 222 Rosewood Drive, Danvers, MA 01923. Payment may also be made electronically through CCC Online at copyright.com. Other copying for republication, resale, advertising or promotion, or any form of systematic or multiple reproduction of any material in this book is prohibited except with permission in writing from the publisher. The CCC fee code is $0277-786 \mathrm{X} / 17 / \$ 18.00$.

Printed in the United States of America.

Publication of record for individual papers is online in the SPIE Digital Library.

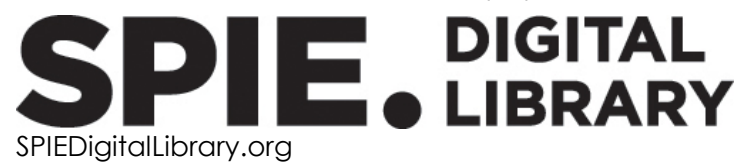

Paper Numbering: Proceedings of SPIE follow an e-First publication model, with papers published first online and then in print. Papers are published as they are submitted and meet publication criteria. A unique citation identifier (CID) number is assigned to each article at the time of the first publication. Utilization of CIDs allows articles to be fully citable as soon as they are published online, and connects the same identifier to all online, print, and electronic versions of the publication. SPIE uses a seven-digit CID article numbering system in which:

- The first five digits correspond to the SPIE volume number.

- The last two digits indicate publication order within the volume using a Base 36 numbering system employing both numerals and letters. These two-number sets start with 00, 01, 02, 03, 04, 05, 06, 07, 08, 09, OA, OB ... 0Z, followed by 10-1Z, 20-2Z, etc.

The CID Number appears on each page of the manuscript. The complete citation is used on the first page, and an abbreviated version on subsequent pages. 


\title{
Contents
}

\author{
vii Authors \\ xi Conference Committee \\ xiii Introduction
}

SESSION 1 DESIGN, DEVELOPMENT, AND FABRICATION OF PHOTONIC INSTRUMENTS I

1011003 Modeling diffractive effects due to micro-lens arrays on liquid crystal panels in projectors [10110-2]

1011004 Automatic correction of diffraction pattern shift in a pushbroom hyperspectral imager with a piezoelectric internal line-scanning unit [10110-3]

1011005 All plastic ultra-small size imaging lens unit fabrication and evaluation for endoscope [10110-4]

1011006 Design and evaluation of a freeform lens by using a method of luminous intensity mapping and a differential equation [10110-5]

\section{SESSION 2 METROLOGY, CHARACTERIZATION, AND FABRICATION OF PHOTONIC INSTRUMENTS}

1011007 Optical stent inspection of surface texture and coating thickness [10110-6]

1011008 Determination of the paraxial focal length using Zernike polynomials over different apertures [10110-7]

1011009 Highly sensitive measurement of submicron waveguides based on Brillouin scattering [10110-8]

$101100 \mathrm{~A}$ Capabilities and challenges in transferring the wavefront-based alignment approach to small aperture multi-element optical systems (Invited Paper) [101 $10-9$ ]

10110 OB System-level analysis and design of a compact RGB-NIR CMOS camera [10110-10]

\section{SESSION 3 APPLICATIONS OF PHOTONIC INSTRUMENTS I}

$101100 C$ Double-sideband filter for digital holography [10110-11]

10110 OD Advances in broadband-integrated optic beam combiners for mid-IR astronomical interferometers [10110-12] 
$101100 \mathrm{OE}$ Locally resolved characterization of progressive addition lenses by calculation of the modulation transfer function using experimental ray tracing [1 $10110-13]$

$10110 \mathrm{OF}$ Optical coherence tomography for non-invasive examination and conservation of cultural heritage objects [10110-14]

\section{SESSION $4 \quad$ APPLICATIONS OF PHOTONIC INSTRUMENTS II}

$10110 \mathrm{OH} \quad$ Hyperspectral calibration method for CMOS-based hyperspectral sensors [10110-17]

1011001 Optical frequency-domain reflectometry using multiple wavelength-swept elements of a DFB laser array [10110-18]

$10110 \mathrm{JJ}$ Optical coating uniformity of $200 \mathrm{~mm}$ (8") diameter precut wafers [10110-19]

\section{SESSION 5 APPLICATIONS OF PHOTONIC INSTRUMENTS III}

10110 OK Ultra-compact imaging plate scanner module using a MEMS mirror and specially designed MPPC [10110-20]

$10110 \mathrm{OL}$ 3D label-free super-resolution imaging [10110-21]

10110 OM Round Robin test on bio-imaging transfer standard for 3D optical profilers [10110-22]

1011000 Development of a high-throughput solution for crystallinity measurement using THz-Raman spectroscopy [10110-24]

\section{SESSION 6 APPLICATIONS OF PHOTONIC INSTRUMENTS IV}

10110 OP Multi-wavelength mid-IR light source for gas sensing [10110-25]

$101100 Q$ Stable wavelength-swept light source designed for industrial applications using KTN beamscanning technology [10110-26]

10110 OR Layer by layer: complex analysis with OCT technology [10110-27]

10110 OS Innovative polarization-holographic imaging Stokes spectropolarimeter for astronomy [10110-15]

\section{SESSION 7 SENSORS AND RUGGEDIZED SYSTEMS I}

101100 Design of a photonic integrated based optical interrogator [10110-29]

$101100 \mathrm{~V}$ Torsion sensing setup based on a Mach-Zehnder interferometer with photonics crystal fiber [10110-30]

$101100 X$ Gain-assisted broadband ring cavity enhanced spectroscopy [10110-32] 
$101100 Z$ A quantitative comparison of dispersion- and absorption-spectroscopic gas sensing [101 10-34]

1011011 Using quantum-dots to enable deep-UV sensitivity with standard silicon-based imaging detectors [10110-36]

SESSION 9 DESIGN, DEVELOPMENT, AND FABRICATION OF PHOTONIC INSTRUMENTS II

1011012 Snapshot Stokes polarimeters based on a single biaxial crystal [10110-37]

1011014 Fast and compact internal scanning CMOS-based hyperspectral camera: the Snapscan [101 10-39]

1011015 Evaluation of the thermal stability of a low-coherence interferometer for precision surface profilometry [10110-40]

1011016 Development of an integrated sub-picometric SWIFTS-based wavelength meter [10110-41]

SESSION 10 DESIGN, DEVELOPMENT, AND FABRICATION OF PHOTONIC INSTRUMENTS III

1011018 Quantum-cascade-laser-based heterodyne phase-sensitive dispersion spectroscopy in the mid-IR range: capabilities and limitations [10110-43]

1011019 Miniature Raman spectroscopy utilizing stabilized diode lasers and 2D CMOS detector arrays [10110-44]

POSTER SESSION

$101101 \mathrm{C}$ A pH sensing system using fluorescence-based fibre optical sensor capable of small volume sample measurement [10110-49]

10110 1D Lens-free imaging-based low-cost microsensor for in-line wear debris detection in lube oils [101 10-50]

$101101 \mathrm{~F} \quad$ Multimodal backside imaging of a microcontroller using confocal laser scanning and optical-beam-induced current imaging [10110-53]

$101101 G \quad$ Fiber-optic-based interferometric sensor [10110-54]

$101101 \mathrm{H} \quad$ High-resolution fast temperature mapping of a gas turbine combustor simulator with femtosecond infrared laser written fiber Bragg gratings [10110-55]

$101101 \mathrm{~J} \quad$ Phase demodulation of Fabry-Perot interferometer-based acoustic sensor utilizing tunable filter with two quadrature wavelengths [10110-57] 
$101101 \mathrm{~K}$ A flexible fiber displacement sensor with tunable resolution and dynamic range based on a few-mode fiber loop [10110-58]

$101101 \mathrm{~L}$ A spectroscopic method of determining color of petroleum products using CIELab color space with LED illumination [10110-60]

10110 1P Miniature and micro spectrometers market: who is going to catch the value? [10110-64]

$101101 Q$ Automatic optical inspection of regular grid patterns with an inspection camera used below the Shannon-Nyquist criterion for optical resolution [10110-65]

10110 IR Optimization of linear-logarithmic CMOS image sensor using a photogate and a cascode MOSFET for reducing pixel response variation [10110-66]

10110 is Extraction of depth information for 3D imaging using pixel aperture technique [10110-67] 


\section{Authors}

Numbers in the index correspond to the last two digits of the seven-digit citation identifier (CID) article numbering system used in Proceedings of SPIE. The first five digits reflect the volume number. Base 36 numbering is employed for the last two digits and indicates the order of articles within the volume. Numbers start with 00, 01, 02, 03, 04, 05, 06, 07, 08, 09, OA, OB...0Z, followed by 10-12, 20-2Z, etc.

\author{
Abdo, Mohammad, 04 \\ Acedo, Pablo, 0Z, 18 \\ Adib, George A., OX \\ Alajoki, Teemu, OP \\ Artigas, Roger, 07, 0M \\ Assmann, H., 15 \\ Auz, Bryan, 19, 1L \\ Ayoub, Ahmad B., $1 \mathrm{G}$ \\ Badilita, Vlad, 04 \\ Bae, Myunghan, 1R, is \\ Bahl, Mayank, 03 \\ Baselt, T., 15 \\ Belsley, Michael S., 1Q \\ Bermudez, Carlos, 07, 0M \\ Beugnot, Jean-Charles, 09 \\ Bhaskaran, Suraj, 11 \\ Binkele, Tobias, 08 \\ Bohnert, Patrick, 04 \\ Bonneville, Christophe, 16 \\ Bonvallet, Joseph, 19 \\ Bouyé, Clémentine, 1P \\ Brenner, Carsten, IF \\ Bret, Boris P. J., $1 Q$ \\ Brown, Dean, 0J \\ Brunner, Robert, 04 \\ Buczyński, Ryszard, OP \\ Burt, Travis C., OJ \\ Cadevall, Cristina, 07 \\ Campos, Juan, 0C, 12 \\ Chang, Seunghyuk, is \\ Charbonneau, Michel, $1 \mathrm{H}$ \\ Charle, Wouter, 14 \\ Cherchi, Matteo, OP \\ Choi, Byoung-Soo, 1R, is \\ Comstock, Matthew, IL \\ Cooper, Denver, OF \\ Coulas, David, $1 \mathrm{H}$ \\ Deng, Shijie, 1C \\ d'Humières, Benoît, $1 \mathrm{P}$ \\ Diener, Romina, OD \\ DiLazaro, Tom, 이 \\ Ding, Huimin, $1 \mathrm{H}$ \\ Duchemin, Céline, 16 \\ Essameldin, Mahmoud, 06 \\ Estévez, Irene, 12 \\ Estudillo-Ayala, Julian M., OV \\ Farnan, Martin, OU \\ Felgueiras, Paulo E. R., 1Q \\ Fernandes, Filipe, 00
}

Ferreira, Flávio P., $1 Q$

Finkeldey, Markus, $1 \mathrm{~F}$

Fisher, Mark, OJ

Fleischmann, Friedrich, 06, 08, OE

Florin, Christian, OR

Förster, Erik, 04

Forte, Paulo M. F., 1Q

Fosse, Jean-Charles, 00

Fu, Xin, $1 \mathrm{~K}$

Fujimoto, Masatoshi, $\mathrm{OK}, \mathrm{OQ}$

Gallegos-Arellano, E., OV

Gan, Qiaoqiang, $1 G$

García Pérez, A., OM

Geelen, Bert, OB

Gerhardt, Nils C., $1 F$

Godet, Adrien, 09

Gonthiez, Thierry, 16

Goossens, Thomas, $\mathrm{OH}$

Göring, Lena, $1 \mathrm{~F}$

Gorritxategi, Eneko, 1D

Greiner, A., 15

Grundström, Kim, OL

Guina, Mircea, OP

Gutierrez, Gustavo, OE

Haeggström, Edward, OL, OM

Harjanne, Mikko, OP

Hartmann, P., 15

Hayden, Jakob, 0Z, 18

Heinilehto, Noora, OP

Henning, Thomas, 06, 08, OE

Hernandez, Emmanuel, OV

Hernandez-Garcia, J. C., OV

Herrmann, Daniel, 03

Hilbig, David, 08, OE

Ho, Lawrence, 00

Hofmann, Martin, IF

Hou, Isabella, OF

Huang, Qiongdan, OF

Huyet, Guillaume, $1 \mathrm{C}$

lbañez, Sergi, 07

Ibrahim, Selwan K., OU

Ichiyama, Robert, 11

lemmi, Claudio, OC

Imai, Tadayuki, $0 Q$

Ishii, Kenta, 05

Jacobsen, Jake, 03

Jauregui-Vazquez, D., OV

Jayapala, Murali, OB

Jiang, Shibin, $1 \mathrm{~K}$ 
Kakauridze, George, OS

Kalinowski, Przemyslaw, OP

Karabacak, Devrez M., OU

Karioja, Pentti, OP

Kassamakov, Ivan, OL, OM

Kasztelanic, Rafał, OP

Kawai, Shinsuke, 05

Khalil, Diaa, OX

Kilosanidze, Barbara, OS

Kim, Sang-Hwan, IR, IS

Kitagawa, Seiichiro, 05

Kluczyński, Paweł, OP

Koch, E., 15

Korvink, Jan G., 04

Krappig, Reik, OA

Kujawa, Ireneusz, OP

Kurkhuli, Georgi, OS

Kvernadze, Teimuraz, OS

Labadie, Lucas, OD

Laguarta, Ferran, 07

Lambrechts, Andy, OB, OH, 14

Lang, Walter, 06

Lee, Jimin, 1R, 1S

Lee, Sang-Jin, is

Lendl, Bernhard, $0 Z, 18$

Lewis, Liam, $1 \mathrm{C}$

Liao, Hao, $1 \mathrm{~J}$

Liu, Deming, $1 \mathrm{~J}, 1 \mathrm{~K}$

Liv, Li, $1 \mathrm{~J}$

Liu, Xuan, OF

Lizana, Angel, OC, 12

LU, Ping, $1 \mathrm{~J}, 1 \mathrm{~K}$

Mabe, Jon, 1D

Martin, Bruno, 16

Martín-Mateos, Pedro, 0Z, 18

Matilla, Aitor, 07

McAuliffe, Michael A. P., 1C

Mihailov, Stephen J., $1 \mathrm{H}$

Minardi, Stefano, OD

Miyamoto, Yuichi, OK

Morino, Eric, 16

Nader, Abd El-Rahman, $1 G$

Ndao, Abdoulaye, 09

Nehmetallah, Georges, 0

Nelsen, B., 15

Ninkov, Zoran, 11

Nishihara, Atsuhiko, 05

Nolte, Stefan, OD

Nolvi, Anton, OL, OM

Nunes-Pereira, Eduardo J., $1 Q$

Oh, Chang-Woo, 1R, 1S

Okamoto, Dai, 05

Oliveres, Robin, 16

Ollila, Jyrki, OP

Olmstead, Ty, 19, $1 \mathrm{~L}$

Pacheco-Chacon, Eliana I., OV

Park, JongHo, is

Patel, Divya, OF

Peinado, Alba, 12

Phan Huy, Kien, 09
Pichette, Julien, $\mathrm{OH}, 14$

Puget, Renaud, 16

Ramachandran, Nanthan, $1 \mathrm{H}$

Ramírez, Claudio, 0C, 12

Ratajczyk, Marcin, OP

Ringwald, Alexandre, 00

Robinson, Ross, 11

Rodriguez, John D., 19, $1 \mathrm{~L}$

Rojas-Laguna, Roberto, OV

Roy, Anjan, 00

Saad, Mai, $1 G$

Sabry, Yasser M., OX

Sagberg, Håkon, $\mathrm{OP}$

Sakamoto, Tadashi, $\mathrm{OQ}$

Sakamoto, Takashi, $0 Q$

Salaj-Kosla, Urszula, 1C

Salo, Tomi, OP

Sandler, N., OM

Sasaki, Kensuke, OK

Sasaki, Yuzo, OQ

Schellenberg, Falk, IF

Schmitt, Robert, OA

Selim, Mahmoud A., OX

Shin, Jang-Kyoo, IR, IS

Sierra-Hernandez, Juan M., OV

Sopo, Victor, 12

Spooren, Nick, OB

Stürmer, Moritz, 04

Sugai, Eiichi, $O Q$

Suomalainen, Soile, OP

Swillam, Mohamed, IG

Sylvestre, Thibaut, 09

Tack, Klaas, OB

Tai, Hidetoshi, 05

Takasaka, Masaomi, OK

Taudt, Ch., 15

Tepper, Jan, OD

Thomas, Fabrice, 16

Thomson, Robert R., OD

Tokuda, Kimio, 05

Toyoda, Seiji, OQ

Troiani, David, OJ

Turpin, Alex, 12

Ueno, Masahiro, $0 Q$

Ushio, Makoto, 05

Valognes, Nicolas, 16

Viheriälä, Jukka, OP

Viitala, T., OM

Virtanen, Sami, OP

Vunckx, Kathleen, $\mathrm{OH}$

Walker, Robert B., $1 \mathrm{H}$

Wallrabe, Ulrike, 04

Williams, Scott, 11

Wolfe, Raymond, 1C

$\mathrm{XU}$, Chenglin, 03

Yagi, Shogo, $0 \mathrm{Q}$

Yamada, Mahiro, $0 Q$

Yamaguchi, Joji, $0 Q$

Yamamoto, Koei, OK, OQ

Yang, Yi, OF 
Yun, Sangsig, $1 \mathrm{H}$

Zaki, Farzana, OF

Zhang, Jiangshan, $1 \mathrm{~J}, 1 \mathrm{~K}$

Zia, Nouman, OP

Zubia, Joseba, 1D

Proc. of SPIE Vol. $101101011001-9$

Downloaded From: https://www.spiedigitallibrary.org/conference-proceedings-of-spie on 26 Apr 2023 Terms of Use: https://www.spiedigitallibrary.org/terms-of-use 
Proc. of SPIE Vol. 10110 1011001-10

Downloaded From: https://www.spiedigitallibrary.org/conference-proceedings-of-spie on 26 Apr 2023 Terms of Use: https://www.spiedigitallibrary.org/terms-of-use 


\section{Conference Committee}

Symposium Chairs

Jean-Emmanuel Broquin, IMEP-LAHC (France)

Shibin Jiang, AdValue Photonics, Inc. (United States)

Symposium Co-chairs

Connie J. Chang-Hasnain, University of California, Berkeley

(United States)

Graham T. Reed, Optoelectronics Research Centre, University of

Southampton (United Kingdom)

Program Track Chair

Yakov Sidorin, Quarles \& Brady LLP (United States)

Conference Chair

Yakov G. Soskind, DHPC Technologies (United States)

Conference Co-chair

Craig Olson, L-3 Communications (United States)

\section{Conference Program Committee}

Daniel Asoubar, LightTrans International UG (Germany)

James B. Breckinridge, California Institute of Technology

(United States)

Lynda E. Busse, U.S. Naval Research Laboratory (United States)

James T. A. Carriere, Ondax, Inc. (United States)

John D. Corless, Verity Instruments, Inc. (United States)

David G. Fischer, NASA Glenn Research Center (United States)

Chris Hessenius, College of Optical Sciences, The University of Arizona

(United States)

Gary B. Hughes, California Polytechnic State Univ., San Luis Obispo

(United States)

Filipp V. Ignatovich, Lumetrics, Inc. (United States)

Jacob B. Khurgin, Johns Hopkins University (United States)

Matthew P. Lumb, U.S. Naval Research Laboratory (United States)

Patrick C. Mock, Ziva Corporation (United States)

Darryl Naidoo, CSIR National Laser Center (South Africa)

Nada A. O'Brien, Oculus Research (United States)

Jeff Throckmorton, Avo Photonics, Inc. (United States)

Mariano Troccoli, AdTech Optics, Inc. (United States) 


\section{Session Chairs}

1 Design, Development, and Fabrication of Photonic Instruments I

Yakov G. Soskind, DHPC Technologies (United States)

2 Metrology, Characterization, and Fabrication of Photonic Instruments

Yakov G. Soskind, DHPC Technologies (United States)

3 Applications of Photonic Instruments I

Nada A. O'Brien, Oculus Research (United States)

4 Applications of Photonic Instruments II

Nada A. O'Brien, Oculus Research (United States)

5 Applications of Photonic Instruments III

Daniel Asoubar, LightTrans International UG (Germany)

6 Applications of Photonic Instruments IV

Daniel Asoubar, LightTrans International UG (Germany)

7 Sensors and Ruggedized Systems I

James T. A. Carriere, Ondax, Inc. (United States)

8 Sensors and Ruggedized Systems II

James T. A. Carriere, Ondax, Inc. (United States)

9 Design, Development, and Fabrication of Photonic Instruments II Jeff Throckmorton, Avo Photonics, Inc. (United States)

10 Design, Development, and Fabrication of Photonic Instruments III Jeff Throckmorton, Avo Photonics, Inc. (United States) 


\section{Introduction}

As photonics-based solutions continue to deepen our fundamental knowledge, enhance our ability to interact with each other, and extend our chances of living longer, healthier lives, Photonic Instrumentation Engineering conference is continuing to see a significant expansion in the numbers of submitted papers and presented talks.

We encourage you, the reader, to actively participate in this annual event by presenting innovative results of your work, and by attending the conference presentations of your choice.

Yakov G. Soskind Craig Olson 
Proc. of SPIE Vol. 10110 1011001-14

Downloaded From: https://www.spiedigitallibrary.org/conference-proceedings-of-spie on 26 Apr 2023 Terms of Use: https://www.spiedigitallibrary.org/terms-of-use 